\title{
Progress and challenges for malaria vaccines
}

\author{
Thomas L. Richie ${ }^{\star}$ Allan Saul $\dagger$ \\ ${ }^{*}$ Malaria Program, Naval Medical Research Center, 503 Robert Grant Avenue, Silver Spring, Maryland 20910-7500, USA \\ (e-mail: richiet@nmrc.navy.mil) \\ $\dagger$ Malaria Vaccine Development Unit, NIAID, National Institutes of Health, Twinbrook I, 5640 Fisher Lane, Rockville, Maryland 20853, USA \\ (e-mailasaul@niaid.nih.gov)
}

\begin{abstract}
Malaria causes much physical and economic hardship in tropical regions, particularly in communities where medical care is rudimentary. Should a vaccine be developed, it is the residents of these areas that stand to benefit the most. But the vaccine, which has been promised to be 'just round the corner' for many years, remains elusive. It is important to ask why this is so, when effective vaccines exist for many other infectious diseases. What are the reasons for the slow rate of progress, and what has been learned from the first clinical trials of candidate malaria vaccines? What are the remaining challenges, and what strategies can be pursued to address them?
\end{abstract}

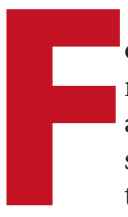
our species of malaria infect humans, which raises an initial question of how many vaccines are needed. Studies in the 1950-60s of sequential heterologous infections in malaria therapy patients, such as Plasmodium vivax after Plasmodium falciparum ${ }^{1}$, and cross-species challenge experiments in the 1970s using the irradiated sporozoite vaccine $^{2}$ showed that protection against several species will be difficult to achieve with a single vaccine. Accordingly, species-specific vaccines for P. falciparum and $P$. vivax - the two parasites that contribute most heavily to the malaria burden — are being developed, keeping open the possibility of combining the antigens into a single formulation sometime in the future. Once successful vaccines have been developed against $P$. falciparum and $P$. vivax, it should be relatively straightforward to create similar vaccines for Plasmodium malariae and Plasmodium ovale.

The P. falciparum parasite deserves particular attention because of the variety and severity of disease syndromes that it causes (see review in this issue by Miller et al., pages 673-679). Several risk groups are found among those living in endemic areas who are subject to repeated $P$. falciparum infections: infants and young children suffer particularly from life-threatening anaemia, older children from an induced $\mathrm{coma}^{3}$, and primagravida women from severe disease related to placental sequestration ${ }^{4}$. For each of these groups, an anti-morbidity vaccine, possibly tailored to the underlying pathophysiology, would be of great benefit. Malaria-naive travellers, either crossing international borders or travelling from malaria-free to malaria-endemic areas in their own countries, constitute another risk group, and are susceptible to severe disease after acquiring their first infection; for this group, it is important to prevent malaria infection altogether.

Although practical considerations of both development and production costs favour a single vaccine for $P$. falciparum, the different risk groups and vaccine requirements have generated at least three approaches for this species alone (Box 1): an anti-infection vaccine aimed at protecting malaria-naive travellers or residents of low endemic areas from becoming infected; an anti-disease/anti-mortality vaccine aimed at children, pregnant women and migrants living in endemic areas; and an anti-mosquito-stage vaccine aimed at preventing the transmission of malaria from one person to another ${ }^{5}$.

In this review, we discuss the challenges faced in developing these vaccines, why we believe that success is likely, the strategies being pursued and the progress to date, including some encouraging results from clinical trials. We outline the tasks that remain and how they are being addressed by a worldwide effort.

\section{Attacking a chronic infection}

To appreciate the constraints of curtailing malaria infection with a vaccine, consider that the parasite that can establish a chronic infection in an immunocompetent host, successfully evading all branches of the immune system. This contrasts with most acute infectious diseases, for which it is relatively straightforward to reproduce the sterile immunity that follows natural infection. There are several immune responses that restrict parasite growth, but the parasite persists. Indeed, the parasite benefits from immune responses if they lead to chronic infection and thereby enhance transmission to the mosquito. As yet, none of the immune responses identified in humans robustly predicts protection from infection or from disease, nor do we know for sure which branches of the immune system will succeed ultimately in eradicating the parasite. Thus, the plan to harness immune responses to destroy the parasite presents a conundrum and requires clever approaches that short-circuit the mechanisms of immune evasion.

Complicating the picture, the different stages of the parasite express different antigens, and a vaccine effective in killing liver-stage parasites may not inhibit the growth of blood-stage parasites. Many parasite proteins exhibit polymorphism, which potentially limits the effectiveness of any vaccine not incorporating distinct variants of antigen. For example, a single parasite clone contains roughly 50 different copies of the gene for the variable surface antigen PfEMP1 (P. falciparum erythrocyte membrane protein 1). During a chronic infection, each successive wave of parasitaemia expresses a new variant surface antigen, thus 


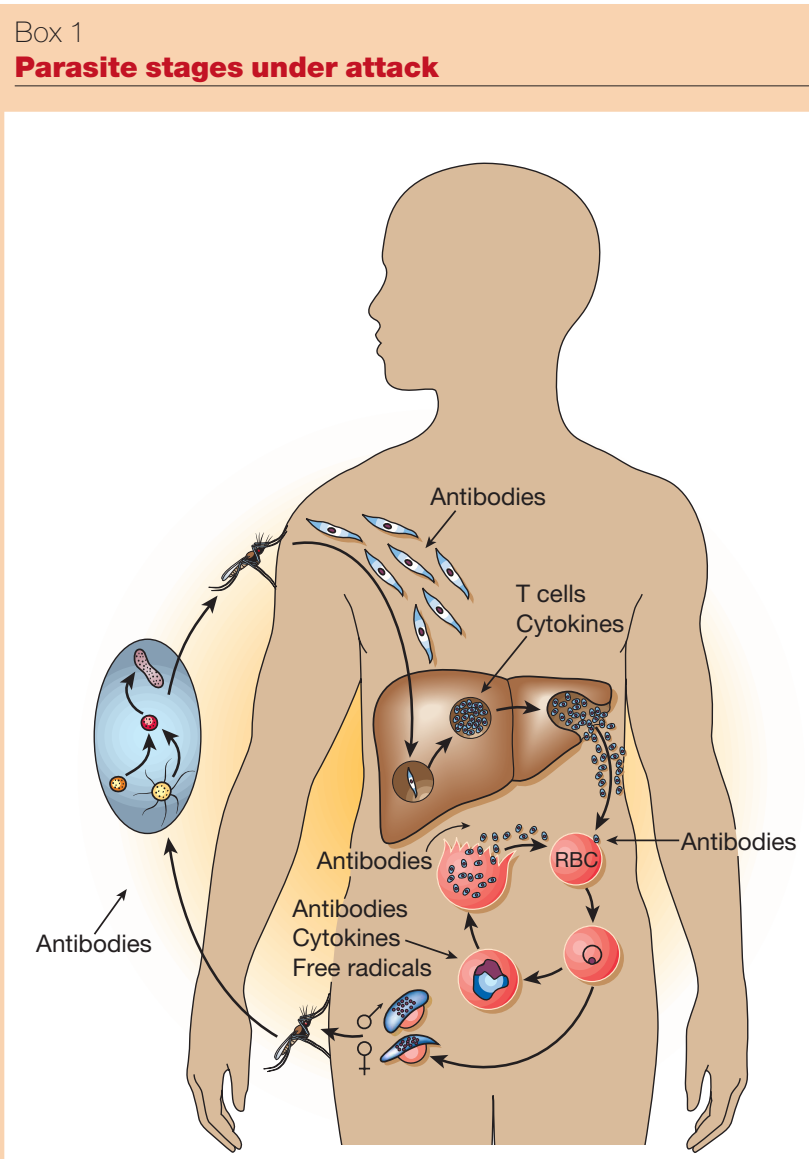

Current approaches to malaria vaccine development can be classified according to the parasite stages that are targeted: 1. Vaccines directed against sporozoites and/or liver stages (collectively termed pre-erythrocytic stages) are designed to prevent blood-stage infection and thereby avoid all manifestations of disease (anti-infection vaccines).

2. Vaccines directed against asexual blood stages are designed to reduce clinical severity (anti-morbidity/mortality vaccines).

3. Vaccines directed against mosquito stages are designed to halt development in the mosquito (transmission-blocking vaccines). Protective mechanisms of immunity are shown for each stage.

In reality, the effects that can be anticipated for each type of vaccine overlap broadly: a pre-erythrocytic-stage vaccine, even if not $100 \%$ effective, could reduce transmission and morbidity - the latter is predicted by the reductions in morbidity and mortality associated with the use of insecticide-impregnated bednets; a highly effective blood-stage vaccine could eliminate blood stages as soon as they emerge from the liver, thereby curtailing both infection and transmission; and a transmission-blocking vaccine could reduce population-wide malaria infection rates and malariaassociated morbidity.

allowing parasite multiplication despite the presence of antibodies directed against the preceding parasitic wave ${ }^{6}$. The sheer number of malaria proteins - estimated to be at least 5,000-6,000 — presents a perplexing choice for the designer of a subunit vaccine (see review in this issue by Hoffman et al., pages 702-709). On the other side of the parasite-host equation, humans exhibit striking heterogeneity in their immune response, depending on their type of human leukocyte antigen (HLA) and other factors (Fig. 1). There is also a bewildering array of other genetic traits, such as haemoglobin type and red cell polymorphisms, that control susceptibility to and have been

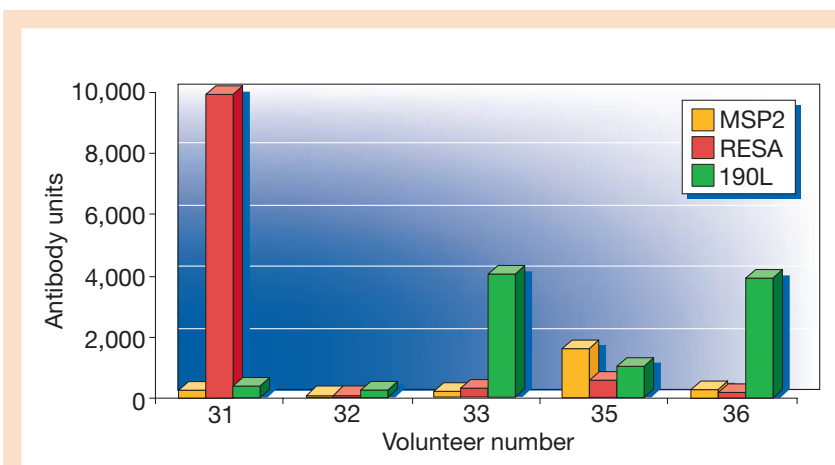

Figure 1 Diversity of immune responses in humans to malaria antigens. Data from five consecutive volunteers (volunteer numbers $31-36 ; 34$ was a placebo) receiving a mixture of $50 \mu \mathrm{g}$ of each of three malaria antigens, the $190 \mathrm{~L}$ fragment of MSP1, MSP2 and RESA, in Montanide ISA720, followed by a boost of $20 \mu \mathrm{g}$ of each antigen $^{30}$. A similar diversity of responses was seen in volunteers who received the three antigens separately. Tasks for vaccine developers include increasing the immunogenicity of malaria antigens through new formulations and approaches, and designing vaccines that induce protective immune responses in everyone, despite diverse genetic backgrounds.

selected by malaria ${ }^{7}$. Given these complexities, is it possible to make an effective vaccine?

\section{Why a vaccine is feasible}

Several studies have shown that malaria vaccines are feasible. First, immunization with irradiated sporozoites protects or partially protects rodents ${ }^{8}$, monkeys ${ }^{9}$ and humans ${ }^{2,10,11}$ from being infected by sporozoites. In humans, immunization through the bites of more than 1,000 irradiated, infected mosquitoes, in batches of a few hundred bites over several months, confers sterilizing protection against sporozoite challenge in more than $90 \%$ of vaccine recipients. Although the immune mechanisms underlying this protection are not known, evidence indicates the importance of T-cell responses directed against parasite proteins expressed on the surface of infected hepatocytes ${ }^{12}$.

So far we have not identified a single dominant immune response in irradiated sporozoite-immunized hosts ${ }^{13}$. This suggests, but does not establish, that protection may be mediated by the summation of many modest immune responses against the large variety of antigens presented by this attenuated-organism vaccine. Although irradiated sporozoites delivered by mosquito bite are unlikely to be a practical vaccine, their effectiveness indicates that a multivalent pre-erythrocytic (sporozoite and liver)-stage vaccine might induce sterile immunity. This type of anti-infection vaccine would prevent all manifestations of disease and would be especially suited for travellers or others exposed to relatively low intensity transmission, but may also have an important role in limiting malaria-related morbidity and mortality in those living in endemic areas.

Second, people infected repeatedly by malaria develop 'naturally acquired immunity' (NAI), which protects against clinical disease ${ }^{14}$. But if their parasites are eliminated through radical drug cure, these individuals can become re-infected, which indicates that NAI does not include absolute anti-infection immunity ${ }^{15}$. If we could reproduce NAI with a vaccine (that is, use a vaccine to hasten the transition from malaria-naive to clinically immune), we could shelter recipients from malarial disease. Antibody responses to blood-stage parasites constitute part of NAI, because passive transfer of purified immunoglobulins from immune individuals protects children $^{16-18}$. Thus, another avenue for designing a vaccine is to generate antibody responses to blood-stage parasite antigens. This type of vaccine, which would suppress blood-stage parasites and prevent disease, would be especially suited to those exposed to intense 
transmission and could be used either alone or as a complement to a pre-erythrocytic-stage vaccine. The efficacy of such a vaccine for particular target groups might be improved by tailoring its composition to the underlying pathophysiology, such as the inclusion of components that block placental sequestration in a vaccine designed for women considering pregnancy.

Third, immunization studies show that vaccines already in hand can protect against malaria infection in animals models and in humans. Notably, consistent protection against experimental sporozoite challenge has been achieved in naive humans vaccinated with a recombinant anti-sporozoite vaccine based on the circumsporozoite protein - the principal surface antigen of sporozoites ${ }^{19}$. Protection has been achieved routinely in new world monkeys (Aotus and Saimiri) against blood-stage challenge with $P$. falciparum by vaccination with the antigen merozoite surface protein $1(\mathrm{MSP} 1)^{20}$. Both types of vaccine, anti-sporozoite and anti-blood-stage, have provided evidence of protection in field trials (see below), although the efficacy of these vaccines is still too low and the duration of protection still too short to be of practical value.

Last, studies have shown success in protecting mosquitoes against infection by $P$. falciparum and $P$. vivax. In these studies, the gametocyte-containing blood that is used as the infection source for the mosquito is mixed with serum from animals immunized with prototype human anti-mosquito-stage (transmission-blocking) vaccines ${ }^{5,21}$. Thus, the efficacy of all three types of vaccine against human parasites - aimed at the sporozoite stage, blood stage and mosquito stage has been proved in principle.

\section{Strategies for vaccine design}

Given that there is evidence supporting the feasibility of all three types of vaccine, what is the best strategy for designing them? What antigens should be included, and how should they be formulated? Certain principles can be defined that guide development.

First, we should choose antigens that meet specific criteria. Antigens are favoured as candidates if they are accessible to the immune system, induce protective immune responses in animal models, and either lack antigenic diversity or have at least limited diversity. Antigens such as PfEMP1, which show considerable diversity but are potentially outstanding vaccine targets, will require a means by which to overcome that diversity - for example, by focusing on conserved functional domains ${ }^{22}$. Parasite receptors that mediate the binding of merozoites to erythrocytes, or of infected cells to endothelial cells, are important candidates. Some of these interactions are regulated by redundant pathways that may need to be blocked simultaneously (see review by Miller et al., pages 673-679). Consideration of the parasite biology may lead to new antigens, such as proteins that are expressed in the mid-gut of mosquitoes and have therefore not evolved under human immune selection pressure, but which could be the targets of antibodies taken up in the mosquito's blood meal. Current candidate antigens are described in Table 1.

Second, we should combine antigens from different stages. Because of the stage specificity of antigen expression, the escape of even small numbers of parasites into the blood of a non-immune individual after immunization with a pre-erythrocytic-stage vaccine will result in clinical malaria. In recent experiments, naive volunteers challenged with 100-1,000 ring-infected erythrocytes (roughly 30-300-fold fewer than released from a single sporozoite-infected hepatocyte) had unaltered growth rates of the parasite during the pre-patent period and also developed clinical malaria at the expected threshold density of parasitaemia ${ }^{23}$. But if a pre-erythrocytic vaccine were combined with a second line of defence — namely, a component designed to limit the growth of asexual blood stages - then vaccinated individuals with break-through blood-stage infection would be able to reduce further the risk of significant clinical illness. As another example, a combination of an anti-mosquito-stage vaccine with a blood- or pre-erythrocytic-stage vaccine would decrease the probability of resistant clones that emerge in vaccinated hosts spreading through the population.

Third, we should combine several antigens from a single stage. There are several arguments in favour of multivalency. (1) If the protection induced by each antigen is independent of that afforded by its companions, and if each is unable to induce sterile immunity on its own, then the potency of the vaccine will increase with more antigens. Few data support the supposition of independence, however, and problems with interference among antigens, if encountered, would have to be offset by the benefits of including several antigens or

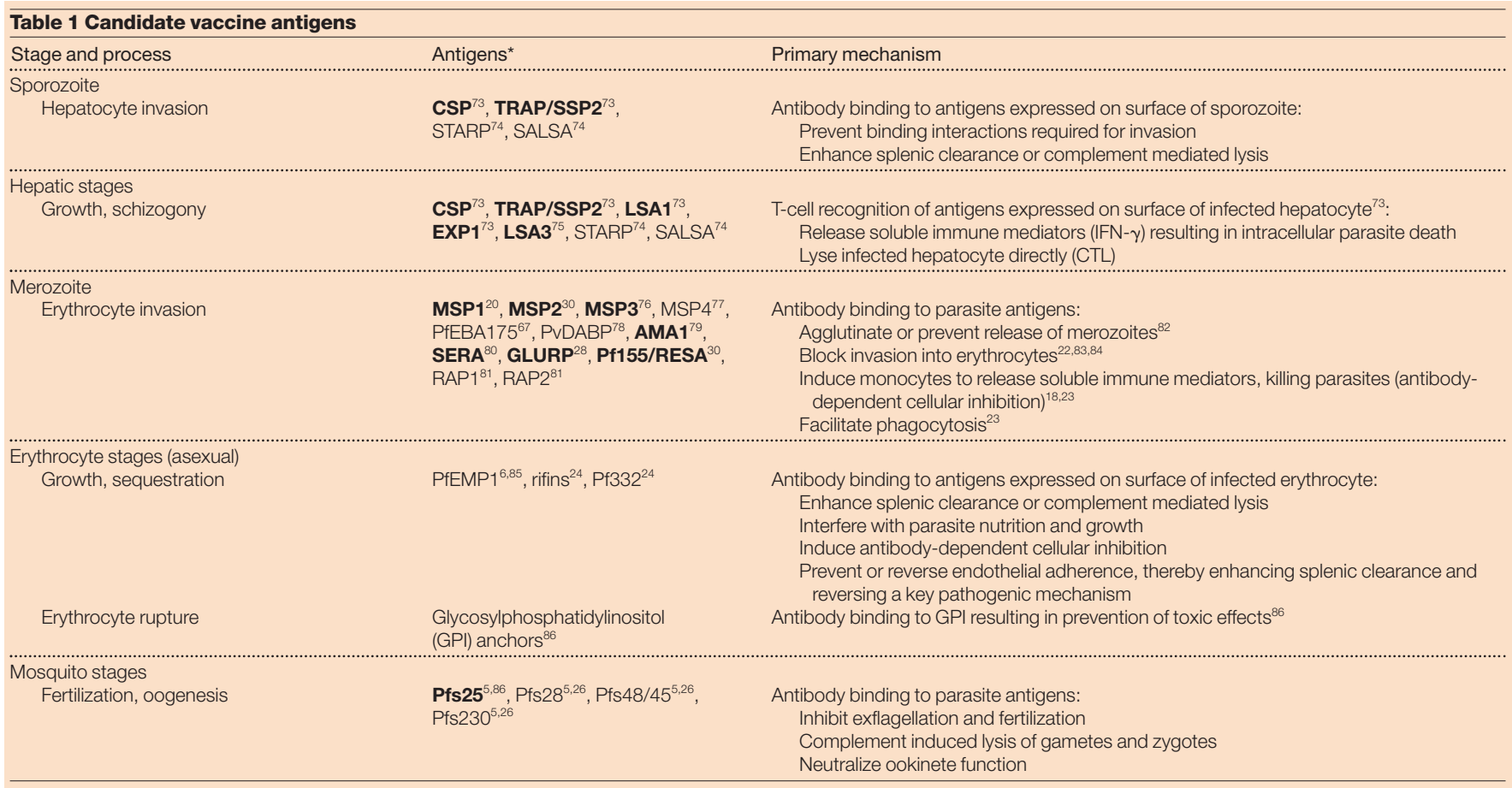

${ }^{*}$ Antigen names in bold type are in clinical development. Antigens have been listed according to the broad category of vaccine in which they are most likely to be included. However, many antigens are expressed in several stages (for example, LSA3 is expressed in blood stages as well as the liver, and most merozoite antigens are probably expressed in liver stages). Thus antigens in one category may also be targets in other stages. 
by alterations to vaccine design that eliminate the interference. (2) Many leading vaccine candidates (MSP1 and apical membrane antigen 1 (AMA1)) are polymorphic, and several forms of each antigen may be required to provide protection against parasite diversity $^{24}$. (3) The immune response developed by each person is unique (owing to polymorphisms in HLA, other genetic traits and previous exposure); therefore mixtures of antigens may be required to ensure that each person has a reasonable chance of generating a sufficient response, particularly for genetically restricted T-cell responses. (4) Combining antigens may facilitate the development of a single vaccine that protects against more than one species of malaria. (5) The emergence of parasites that are simultaneously resistant to all immune responses induced by a multivalent vaccine is less likely than the emergence of parasites that are resistant to a single component, which could potentially prolong the useful life of the vaccine relative to that of a monovalent formulation.

Fourth, we need to keep vaccines as simple as possible. Optimizing the immunogenicity of individual components in a mixture is likely to be difficult, particularly as the complexity increases. For proteinbased vaccines, the total amount of protein that can be injected in one vaccine is limited by the formulation and possibly by the frequency of adverse events; in addition, malaria vaccines will have to be inexpensive and the cost rises quickly with complexity. Vaccines using naked DNA or delivery systems such as replicons offer an attractive way of increasing the antigenic diversity within these constraints.

Last, we must induce the right kind of immune response. Biological characteristics of each parasite stage determine which immune responses are optimal for parasite destruction. For example, CD8-expressing T-cell and/or CD4-expressing T-cell type 1 responses may be essential for eliminating intracellular liver-stage parasites ${ }^{25}$; by contrast, transmission-blocking vaccines must induce high, sustained amounts of antibody to neutralize antigens in the mosquito gut ${ }^{5,26}$. Blood-stage vaccines also must generate high concentrations of antibody for protection, but may also need to induce other effector mechanisms, such as antibody-dependent cellular inhibition ${ }^{27,28}$. Particular adjuvant systems may be needed to drive responses in one direction or the other, and this might constrain the composition of a particular vaccine.

So far, antigen choice has been dominated by the arbitrary order in which antigens have been identified. This is now changing with the sequencing of parasite genomes and an increasing understanding of parasite biology. We view the sequencing of the P. falciparum genome as a great boon to vaccine developers, because it should allow selection of the very best candidate antigens. Methods for screening the hundreds of proteins expressed during a particular parasite stage for those that induce protective immune responses are already being developed (see review by Hoffman et al., pages 702-709).

These 'guiding principles' clearly involve compromises between conflicting demands. For example, the need to keep vaccines as simple as possible must be balanced against the need to cover antigenic diversity. Many researchers are proposing that a complex vaccine will be required, because this is the 'safe' assumption. Should one or a few antigens prove to elicit a universal protective response, vaccine development would be accelerated considerably.

\section{Clinical trials of malaria vaccines}

Over the past 15 years, a series of phase $1 / 2$ vaccine trials has been reported using synthetic peptides or recombinant proteins based on malarial antigens. Engers and Godal ${ }^{29}$ report that there have been roughly 40 trials up to 1998 . Most trials were directed against sporozoites or liver stages, in which the use of experimental mosquito challenges allows rapid progress through phase 1 to phase $2 \mathrm{a}$ preliminary efficacy studies. Anti-sporozoite vaccines tested have included completely synthetic peptides, conjugates of synthetic peptide with proteins such as tetanus toxoid to provide T-cell help, recombinant malaria proteins, particle-forming recombinant chimaeric constructs, recombinant viruses, and bacteria- and DNA- based vaccines. Several trials of asexual blood-stage vaccines have used either synthetic peptide conjugates or recombinant proteins, and there has been one trial of a transmission-blocking vaccine recombinant Pfs25 (ref. 26; and unpublished data from National Institutes of Health (NIH) Pfs 25 clinical trial). A recurring theme is the difficulty of obtaining a sufficiently strong and long-lasting immune response in humans even though the same vaccine preparation is often strongly immunogenic in test animals ${ }^{30}$. Various strategies have been designed to overcome this limitation, including the exploration of potent immune-stimulatory conjugates or adjuvants to boost the human response. An interesting approach is the in situ expression of the vaccine through attenuated strains of Salmonella ${ }^{31}$.

Vaccines directed against the circumsporozoite protein covering the sporozoite illustrate the frustrating quest for a consistently protective immune response in humans. Early studies with both recombinant proteins, peptide conjugates and recombinant protein conjugates could elicit anti-circumsporozoite antibody, provided marginal protection in phase 2 a studies but provided no protection in field studies ${ }^{32}$. More recently, collaboration between GlaxoSmithKline and the US Army has produced a chimaeric protein, consisting of a fusion between the circumsporozoite protein and the hepatitis B surface antigen (HbsAg), which is expressed in yeast ${ }^{19}$. In the presence of unmodified HBsAg, the mixture forms typical HBsAg particles (the RTS,S vaccine). Although much more immunogenic than recombinant circumsporozoite protein, RTS,S is still incapable of eliciting a protective response when used with conventional adjuvants, but elicits a protective response in about half of vaccine recipients with the AS02 (formerly SBAS2) experimental oil in water adjuvant, which also contains monophosphoryl lipid A (3D-MPL) and the saponin QS21. Other recent approaches to a circumsporozoite vaccine have used synthetic peptide technology to generate immunogens containing both $\mathrm{T}$ - and B-cell epitopes with an endogenous lipophilic adjuvant ${ }^{33}$.

Only a few $P$. falciparum vaccines have been tested in field trials. This area has been dominated by the synthetic peptide vaccine SPf66, which is absorbed on alum and is directed at blood-stage parasites ${ }^{34}$. Initial results of trials in children and adults residing in relatively low endemic regions of South America and children in a highly endemic area of Tanzania suggested that this vaccine might delay the time to a first clinical malaria episode, but subsequent trials in infants in The Gambia and Tanzania, children in Thailand and adults in Brazil failed to show efficacy. In a recent meta-analysis of nine trials involving 9,800 volunteers, Graves and Gelbrand ${ }^{32}$ concluded that there was no evidence of efficacy for SPf66 in Africa but that it caused a modest reduction of malaria attacks in South America.

Two other vaccines that have recently undergone field trials show promise. In a collaboration with the Medical Research Council (MRC) unit in The Gambia, a trial of the RTS,S vaccine in adult Gambian males resulted in a significant reduction in the rate at which they were infected after vaccination, although the protection was not long-lived ${ }^{35}$. A combination of three asexual blood-stage antigens (the 190L fragment of MSP1, one form of the polymorphic MSP2 antigen and a portion of the RESA or ring-infected erythrocyte surface antigen $)^{30}$ has been tested in children aged 5-9 years in the Wosera district of Papua New Guinea. Designed to determine the parasitological outcome, this trial detected significant decreases in the parasite density and the frequency of parasite episodes greater than 1,000 per microlitre, and a major switch in the MSP2 genotype of parasites ${ }^{36}$.

Although protein-based vaccines are promising and are furthest along the pathway towards malaria vaccine development, alternative technologies such as DNA-based vaccines may prove particularly amenable to multivalent formulations and ultimately less expensive to produce, store and deliver than conventional vaccines (ref. 37; and Box 2). Progress with DNA vaccines has been rapid, and studies have demonstrated the following. (1) Protection against $P$. yoelii 
sporozoite challenge in BALB/c mice immunized with a plasmid encoding the $P$, yoelii circumsporozoite protein in 1994 (ref. 38). (2) Improved protection in mice with a two-plasmid vaccine in 1996 (ref. 39). (3) Complete protective efficacy of DNA prime/poxvirus boost regimens in mice in 1998 (ref. 40). (4) Safety of DNA and induction of genetically restricted, antigen-specific, cytotoxic $\mathrm{T}$ lymphocyte (CTL) responses in humans vaccinated with a plasmid encoding $P$. falciparum circumsporozoite protein in 1998 (refs 41,42). (5) Induction of CD8-expressing and CD4-expressing T-cell-dependent interferon- $\gamma$ responses by ELIspot (enzyme-linked immunospot) assay in humans immunized with the same plasmid in 2001 (ref. 43). (6) Partial protection of rhesus monkeys against P. knowlesi sporozoite challenge with a four-plasmid cocktail boosted by poxvirus constructs encoding the priming malarial antigens in 2001 (ref. 44; and W. O. Rogers, unpublished data). (7) Similar success with $P$. cynomolgi challenge of rhesus monkeys immunized with a epitope string derived from $P$. falciparum fused with the cholera toxin B subunit in 2000 (ref. 45). A DNA vaccine including a string of CTL epitopes from six different $P$. falciparum pre-erythrocytic antigens fused to the full-sequence thrombospondin-related adhesion protein (TRAP) from $P$. falciparum, also administered using a plasmid-prime, poxvirus-boost approach, has been in clinical evaluation in Oxford since 1999 and in The Gambia since September 2000, with encouraging results ${ }^{46}$.

DNA plasmids encoding malaria antigens have generated antibody responses in animal models ${ }^{38,47-50}$, but not in humans as yet ${ }^{41}$. The interferon- $\gamma$ responses shown in humans are appropriate for liver-stage vaccines designed to effect intracellular killing, but are probably insufficient for vaccines designed to neutralize circulating sporozoites or blood-stage parasites. Many new modalities to improve expression and immunogenicity and to extend the breadth of the immune response to DNA-based vaccines are now being tested. These include replacing native genes with synthetic genes using codon frequencies optimized for host expression ${ }^{51}$, adding sequences such as the Fc region of immunoglobulin that increase antigen uptake by dendritic cells ${ }^{52}$, absorbing plasmids onto microparticles ${ }^{53}$ to increase antigen uptake by dendritic cells, particle-mediated gene transfer (gene gun) ${ }^{54}$ or jet injection ${ }^{43}$ to enhance plasmid delivery, and co-stimulation with plasmids encoding cytokines, such as granulocyte/macrophage colony-stimulating factor ${ }^{5-57}$, or other immunostimulatory molecules such as $\mathrm{CpG}$ motifs ${ }^{58}$ or an interleukin-2/immunoglobulin fusion protein ${ }^{59}$.

Of the various approaches, the one that is most potent in improving protection in animal models is heterologous vaccination, in which DNA plasmids are used to prime specific immune responses and virus constructs ${ }^{44,50,54,60}$ or recombinant proteins ${ }^{61}$ are used to boost these responses. Several viral vectors are under development, including avian and mammalian recombinant poxviruses ${ }^{62,63}$, nonreplicating and replicating recombinant adenoviruses ${ }^{64}$, and alphavirus replicons ${ }^{65}$. Clinical studies of DNA priming followed by either recombinant poxvirus or protein boosting are in progress at various centres. Although DNA (plasmid) vaccines on their own are currently not sufficiently immunogenic or protective, if immunogenicity can be improved then DNA-based technologies should allow the development of effective malaria vaccines.

\section{Challenges in vaccine development}

Our progress in identifying antigens and the demonstration that several of these can elicit protective immune responses give us reason for optimism. Nevertheless, a substantial investment of resources is needed to improve the degree and duration of protection and to bring vaccines to licensure. But perceptions concerning the economics of developing, manufacturing and distributing vaccines to a global market that comprises some of the world's poorest people limit our ability to mobilize these resources, especially from the private sector.

Typically, pharmaceuticals have been developed through a process that involves screening large numbers of compounds for

\section{Box 2 \\ Cocktail to soothe tropical fevers?}

DNA plasmid encoding malaria antigen
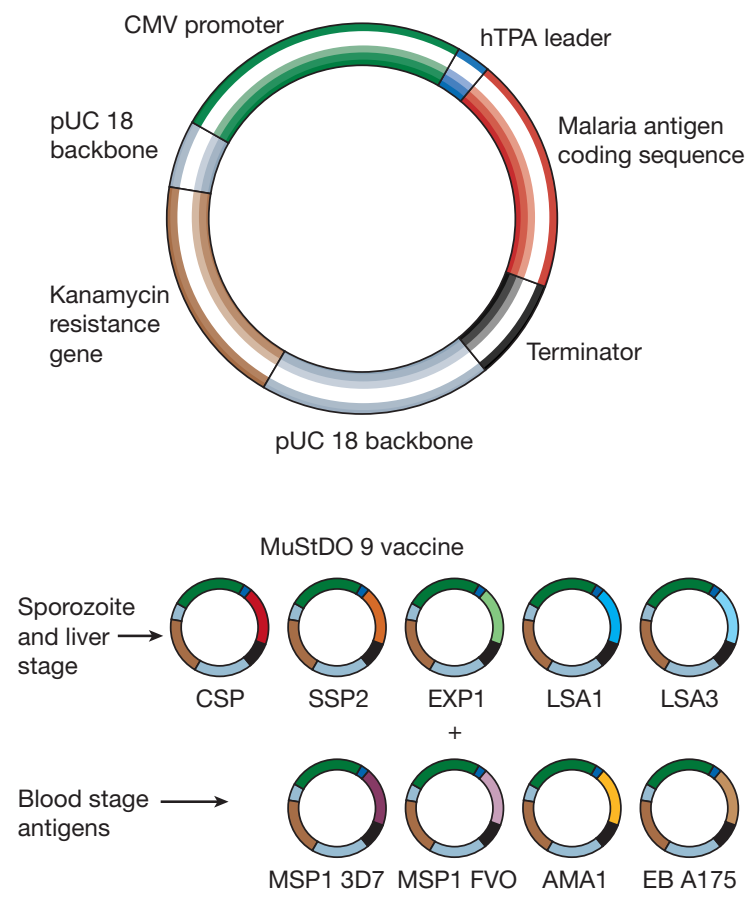

Supercoiled rings of double-stranded DNA containing malaria genes are injected, taken up by muscle cells and translated into malarial proteins. These proteins are processed and presented by antigenpresenting cells, inducing immune responses designed to inhibit the developing malaria parasites. The MuStDO 9 vaccine ${ }^{87,88}$ consists of five plasmids encoding proteins from the sporozoite and liver stages of $P$. falciparum, CSP, SSP2, EXP1, LSA1 and LSA3, and four encoding proteins from blood stages, MSP1 (two alleles representing the major antigenic families), AMA1 and EBA175. The two-tiered design is based on the rationale that parasites escaping the pre-erythrocytic-stage defence will be suppressed by a second line of immune responses directed at blood stages, thus decreasing the likelihood of serious illness in vaccinated individuals with breakthrough blood-stage infections. Each gene in the expression cassette has been synthesized using codon frequencies that are consistent with mammalian patterns. At present, naked DNA vaccines are not sufficiently immunogenic to protect humans against malaria, and require boosting by recombinant viral constructs or recombinant proteins to achieve measurable efficacy. New formulations and designs aiming to increase plasmid uptake by dendritic cells, improve expression and strengthen antibody responses may remove this requirement. So far, DNA plasmids encoding malarial antigens have been administered to roughly 80 healthy adult volunteers in the United States by the US Navy and about 50 healthy adult volunteers in Britain or The Gambia (mainly in heterologous prime-boost regimens with modified vaccinia virus Ankara) by the Oxford University group (A. V. S. Hill, personal communication) and seem to be safe and well tolerated ${ }^{41}$.

activity and picking lead candidates for further development. For malaria we now have at least 5,000 candidate proteins and multiple variants of many of these, and although we have in vitro and animal models that give indications of a candidate's importance, we lack validated models that reliably predict what protection a vaccine formulation will give in humans. Views differ on the contribution 
Box 3

Malaria vaccines in or close to clinical trials

1. Pre-erythrocytic vaccines RTS,S and TRAP/SSP2, and bloodstage vaccines $\mathrm{MSP}_{42}-3 \mathrm{D} 7$ and AMA1-3D7 by a collaboration between the US Army Walter Reed Army Institute of Research (WRAIR), USAID and GlaxoSmithKline.

2. An RTS,S phase 2b trial in Gambian adults and a phase 1 trial in Gambian children being undertaken by the MRC unit in The Gambia, in collaboration with GlaxoSmithKline and with support from WRAIR, the University of Oxford, and The London School of Hygiene and Tropical Medicine.

3. Plasmid DNA, modified vaccinia virus Ankara and an attenuated fowlpox strain encoding the multi-epitope-TRAP insert, by a collaboration between the University of Oxford and the MRC, with phase 1/2a trials in Britain and phase 1 trials in The Gambia ${ }^{46}$. 4. Phase $1 / 2$ a studies of multistage DNA vaccine combinations ('MuStDO') in the United States and Ghana by a collaboration between the US Navy and USAID ${ }^{87,88}$.

5. Phase 1/2a studies of modified vaccinia virus Ankara and a fowlpox strain encoding the circumsporozoite protein by the University of Oxford.
6. Vaccines based on the merozoite surface protein $M S P 1_{19}$ at the Institut Pasteur.

7. Two phase 1 trials by the European Malaria Vaccine Initiative with protein vaccines made by peptide synthesis (MSP3, GLURP), followed by a phase 1 trial of Pichia-expressed recombinant AMA1. 8. Phase 1 studies of $P$. vivax and $P$. falciparum transmission-blocking protein vaccines Pvs25 and Pfs25 by the $\mathrm{NIH}$ Malaria Vaccine Development Unit.

9. Phase 1 studies of a pre-erythrocytic-stage vaccine that is based on a virus-like particle comprising a modified hepatitis $\mathrm{B}$ core particle and circumsporozoite protein-specific T- and B-cell epitopes by a collaboration between Apovia, New York University, Malaria Vaccine Initiative, $\mathrm{NIH}$ and the University of Maryland.

Several other antigens are at an advanced state of development and many phase 1 and some phase 2 clinical trials could reasonably be expected in the next 1-2 years. These include major polymorphisms of AMA1, MSP1 ${ }_{42}$ and MSP2 that explore different expression systems, and single forms of other antigens such as MSP4, RAP2, EBA175 and the P. vivax Duffy-binding protein. that can be made by new and old world primate models to screen candidate vaccines ${ }^{6,67}$. Even where primate models assist antigen selection, the development of useful formulations and vaccine regimens will require several human phase 1 and phase 2 trials, and screening for protection in humans is difficult.

Screening anti-morbidity vaccines in human populations in endemic areas imposes many restraints. First, producing multiple test vaccines is a big task. Second, these vaccines are designed primarily for children, and using children for a primary efficacy screen contributes to the complexity of the trial design. Last, decisions need to be made regarding the end point of the trial. Both for ethical reasons and to diminish sample size requirements, surrogate markers of efficacy may be chosen.

For example, in the Papua New Guinea trial discussed above ${ }^{36}$, parasite density was used as the test of efficacy. This required group sizes of 30 with a 12-week follow up to give reasonable power of detecting a $30 \%$ decrease in parasite density. In the same popula$\operatorname{tion}^{68}$, groups of a few hundred would have been required to see a significant decrease in febrile cases if the vaccine caused a $30 \%$ decrease in malaria-related fever. In this region, the frequency of severe disease or death from malaria is too low to use as a useful end point, even if this could have been justified ethically. In much of Africa - which has an infant mortality of about 100 in 1,000 live births ${ }^{69}$ - depending on the accuracy with which a cause of death can be diagnosed, group sizes of many thousands to tens of thousands would be needed to use mortality as an end point, and this is not feasible at an early stage of vaccine development. Because there are gaps in our understanding of the progression of pathology from parasitaemia to death (see review by Miller et al., pages 673-679), our choice of end point for early efficacy studies is associated with a risk of either discarding a good vaccine because it fails to give an imperfect correlate of protection in early stage testing or wasting scarce resources by taking a poor vaccine through extensive clinical testing.

In contrast to anti-morbidity vaccines for infants or other high risk groups, anti-infection vaccines designed for malaria-naive travellers and residents of low endemic regions face more stringent efficacy constraints, but should be relatively simpler to test. Experimental sporozoite challenge of vaccinated, naive volunteers has become a standard way of early stage testing of vaccines for travellers, especially pre-erythrocytic-stage vaccines ${ }^{70}$, and a bloodstage challenge has also been developed as an alternative for testing blood-stage vaccines ${ }^{23}$.
In principle, mosquito-stage vaccines should have the simplest development path, because there is a relatively straightforward test for assessing the impact of vaccine-induced antibodies on the transmission of parasites to mosquitoes, allowing down-selection to take place at an earlier stage of development ${ }^{26}$. The eventual proof that such a vaccine is effective under field conditions will require large trials in which a high proportion of the population is vaccinated and whole villages are randomized to treatment groups.

\section{Meeting the challenge}

The development of vaccines for the billion people living in endemic countries will require a different scheme from that of more commercially attractive vaccines. The public sector will need to provide the 'push' to facilitate vaccine technology development and early-stage testing and also the 'pull' of guaranteed markets to encourage pharmaceutical firms to license and manufacture vaccines ${ }^{5}$.

The manufacturing capacity to meet global demands may be relatively modest, depending on the type of vaccine. Most of the world's need for a vaccine to prevent serious disease and death in infants will be in sub-Saharan Africa where the live birth rate is 26 million per year. For a recombinant protein vaccine, roughly $1 \mathrm{~kg}$ of each protein component would be needed each year, depending on the number and size of the doses. Even with the non-optimized expression systems that are being used to produce prototype vaccines, this could be met by a single plant capable of fermenting 200 litres per week, which is well within the capacity of biotechnology companies in more industrialized malaria-endemic countries in which national considerations, economics and marketing strategies make local production attractive.

Currently, the biggest bottleneck is the capacity for producing clinical grade material for protein-based vaccines and for performing field trials for all types of vaccine. One of the most encouraging recent developments has been the substantial expansion in government and philanthropic interests to address this need, including the founding of the European Malaria Vaccine Initiative ${ }^{71}$ and the creation of the Malaria Vaccine Initiative at the Program for Appropriate Technology in Health through a grant from the Bill \& Melinda Gates Foundation $^{72}$. The US NIH has developed new programmes for the development and testing of malaria vaccines through its extramural programme and through the creation of an intramural Malaria Vaccine Development Unit. These new developments join several continuing programmes, such as those of the World Health 
Parallel phase 1 testing

of components; combine

after phase 1

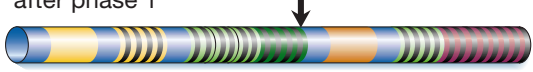

Sequential phase 1 and 2 testing of

components; combine after phase 2

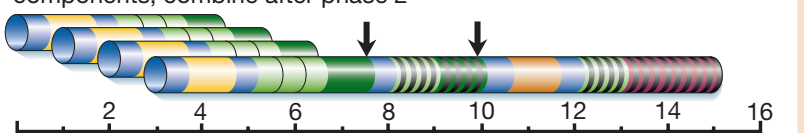

Data review, ethical and

regulatory approval

Phase 1 studies in

developed country

Phase 1 studies in

endemic country

Phase 2 studies in

endemic country

$\square$ Phase 3 manufacture

Phase 3 studies in

endemic country

[ill Combination vaccines

Study may be repeated here. Adds 2-3 years

Figure 2 Timetable of clinical trials. Shown is an optimistic and simplified view of the timetable to completion of phase 3 (pre-registration) trials for a hypothetical tetravalent malaria vaccine starting from the time that clinical grade material is available. Each stage in the process requires review and approval by regulatory bodies. Initial phase 1 safety, immunogenicity and dose escalation testing in adults in the country of manufacture will generally need to be repeated in endemic countries in adults, children and infants, and these trials must run sequentially for safety reasons. The vaccine is then tested for efficacy, and if successful will move to larger phase 3 studies before registration, to confirm its efficacy and safety. Phase 3 trials test the vaccine made under the same conditions that will be used for final manufacture; before they can commence, the final manufacturing process and plant need to be established. Two schemes are examined here. The first combines the four components of the vaccine after the initial phase 1 trial (combination vaccine shown with banding). The second combines the vaccine only after each component is shown to be efficacious. Parallel phase 1 testing takes less time than sequential testing, but requires more resources. Early combination takes fewer resources in field studies, but may require separate studies to show that all components are required (not shown on time line).

Organization, US Agency for International Development (USAID) and the US Department of Defense. Several sites in endemic areas such as The Gambia, Ghana, Kenya, Mali, Mozambique, Papua New Guinea and Tanzania are suitable for vaccine testing.

A potential capacity for vaccine testing has been created through the development of the African Malaria Vaccine Testing Network and its programme of training Africans in clinical trial capability. In addition, many endemic countries are making significant contributions from their scarce resources through the support of staff and facilities for malaria research in general and for vaccines in particular. Despite these developments, progress remains limited by resources, and this limitation will become more acute as vaccine testing programmes gain momentum. Put into perspective, the public support for development of malaria vaccines is roughly tenfold less than the support for a vaccine for HIV - a disease of comparable global impact.

In this setting, several groups have phase $1 / 2$ testing programmes either underway, or have clinical grade immunogens prepared with phase 1 trials commencing in the near future. These developments are summarized in Box 3 .

\section{When will there be malaria vaccines?}

On the assumption that at least some of these vaccines will be efficacious, when can we expect the vaccine? Even under the most optimistic scheme of unlimited resources (Fig. 2), it will still be many years from now, requiring iterative testing of improved combinations and formulations until sufficient efficacy is obtained. In the meantime there is much to do to ensure that when the vaccines are available, health infrastructures are in place to deliver integrated programmes that will use the vaccines effectively. That may be as much of a challenge as the vaccine itself.

1. Jeffery, G. M. Epidemiological significance of repeated infections with homologous and heterologous strains and species of Plasmodium. Bull. World Health Organ. 35, 873-882 (1966).

2. Clyde, D. F. Immunity to falciparum and vivax malaria induced by irradiated sporozoites: a review of the University of Maryland studies, 1971-75. Bull. World Health Organ. 68(Suppl.), 9-12 (1990).

3. Marsh, K. \& Snow, R. W. Malaria transmission and morbidity. Parassitologia 41, 241-246 (1999).

4. Ricke, C. H. et al. Plasma antibodies from malaria-exposed pregnant women recognize variant surface antigens on Plasmodium falciparum-infected erythrocytes in a parity-dependent manner and block parasite adhesion to chondroitin sulfate A. J. Immunol. 165, 3309-3316 (2000).

5. Carter, R., Mendis, K. N., Miller, L. H., Molineaux, L. \& Saul, A. Malaria transmission-blocking vaccines—how can their development be supported? Nature Med. 6, 241-244 (2000).

6. Saul, A. The role of variant surface antigens on malaria-infected red blood cells. Parasitol. Today 15, 455-457 (1999).

7. Miller, L. H. Evolution of the human genome under selective pressure from malaria: applications for control. Parassitologia 41, 77-82 (1999).

8. Nussenzweig, R. S., Vanderberg, J., Most, H. \& Orton, C. Protective immunity produced by the injection of X-irradiated sporozoites of Plasmodium berghei. Nature 216, 160-162 (1967).

9. Collins, W. E. \& Contacos, P. G. Immunization of monkeys against Plasmodium cynomolgi by Xirradiated sporozoites. Nature 236, 176-177 (1972).

10. Egan, J. E. et al. Humoral immune responses in volunteers immunized with irradiated Plasmodium falciparum sporozoites. Am. J. Trop. Med. Hyg. 49, 166-173 (1993).

11. Rieckmann, K. H., Beaudoin, R. L., Cassells, J. S. \& Sell, K. W. Use of attenuated sporozoites in the immunization of human volunteers against falciparum malaria. Bull. World Health Organ. 57(Suppl. 1), 261-265 (1979).

12. Hoffman, S. L. et al. Sporozoite vaccine induces genetically restricted T cell elimination of malaria from hepatocytes. Science 244, 1078-1081 (1989).

13. Hoffman, S.L. et al. Protection of humans against malaria by immunization with radiationattenuated Plasmodium sp. sporozoites. J. Infect. Dis. (in the press).

14. Baird, J. K. Host age as a determinant of naturally acquired immunity to Plasmodium falciparum. Parasitol. Today 11, 105-111 (1995).

15. Hoffman, S. L. et al. Naturally acquired antibodies to sporozoites do not prevent malaria: vaccine development implications. Science 237, 639-642 (1987).

16. Cohen, S., McGregor, I. A. \& Carrington, S. Gamma globulin and acquired immunity to human malaria. Nature 192, 733-737 (1961).

17. Edozien, J. C., Gilles, H. M. \& Udeozo, I. O. K. Adult and cord-blood gamma globulin and immunity to malaria in Nigerians. Lancet ii, 951-955 (1962).

18. Sabchareon, A. et al. Parasitologic and clinical human response to immunoglobulin administration in falciparum malaria. Am. J. Trop. Med. Hyg. 45, 297-308 (1991).

19. Kester, K. E. et al. Efficacy of recombinant circumsporozoite protein vaccine regimens against experimental Plasmodium falciparum malaria. J. Infect. Dis. 183, 640-647 (2001).

20. Stowers, A. W. et al. Efficacy of two alternate vaccines based on Plasmodium falciparum merozoite surface protein 1 in an Aotus challenge trial. Infect. Immun. 69, 1536-1546 (2001).

21. Hisaeda, H. et al. Antibodies to malaria vaccine candidates Pvs 25 and Pvs 28 completely block the ability of Plasmodium vivax to infect mosquitoes. Infect. Immun. 68, 6618-6623 (2000).

22. Gamain, B., Miller, L. H. \& Baruch, D. I. The surface variant antigens of Plasmodium falciparum contain cross-reactive epitopes. Proc. Natl Acad. Sci. USA 98, 2664-2669 (2001).

23. Cheng, Q. et al. Measurement of Plasmodium falciparum growth rate in vivo: a test of malaria vaccines. Am. J. Trop. Med. Hyg. 57, 495-500 (1997).

24. Bolad, A. \& Berzins, K. Antigenic diversity of Plasmodium falciparum and antibody-mediated parasite neutralization. Scand. J. Immunol. 52, 233-239 (2000).

25. Doolan, D. L. \& Hoffman, S. L. The complexity of protective immunity against liver-stage malaria. J. Immunol. 165, 1453-1462 (2000).

26. Carter, R. Transmission blocking malaria vaccines. Vaccine 19, 2309-2314 (2001).

27. Badell, E. et al. Human malaria in immunocompromised mice: an in vivo model to study defense mechanisms against Plasmodium falciparum. J. Exp. Med. 192, 1653-1660 (2000).

28. Oeuvray, C. et al. Cytophilic immunoglobulin responses to Plasmodium falciparum glutamate-rich protein are correlated with protection against clinical malaria in Dielmo, Senegal. Infect. Immun. 68, 2617-2620 (2000)

29. Engers, H. D. \& Godal, T. Malaria vaccine development: current status. Parasitol. Today 14, 56-64 (1998).

30. Saul, A. et al. Human phase I vaccine trials of 3 recombinant asexual stage malaria antigens with Montanide ISA720 adjuvant. Vaccine 17, 3145-3159. (1999).

31. Wu, S. et al. Construction and immunogenicity in mice of attenuated Salmonella typhi expressing Plasmodium falciparum merozoite surface protein 1 (MSP-1) fused to tetanus toxin fragment C. J. Biotechnol. 83, 125-135 (2000).

32. Graves, P. \& Gelbrand, H. Vaccines for preventing malaria. Cochrane Review, The Cochrane Library, issue no. $3<$ http://update-software.com/cochrane/abstracts/ab000129.htm> (Update Software, Oxford, 2001).

33. Nardin, E. H. et al. A totally synthetic polyoxime malaria vaccine containing Plasmodium falciparum $\mathrm{B}$ cell and universal $\mathrm{T}$ cell epitopes elicits immune responses in volunteers of diverse HLA types. $J$ Immunol. 166, 481-489 (2001).

34. Patarroyo, M. E. et al. A synthetic vaccine protects humans against challenge with asexual blood stages of Plasmodium falciparum malaria. Nature 332, 158-161 (1988).

35. Bojang, K. A. et al. Efficacy of RTS,S/ASO2 malaria vaccine against $P$. falciparum infection in semiimmune adult men in The Gambia: a randomised trial. Lancet 358, 1927-1934 (2001).

36. Genton, B. et al. A recombinant blood-stage malaria vaccine reduces Plasmodium falciparum density and exerts selective pressure on parasite populations in a Phase I/IIb trial in Papua New Guinea. J. Infect. Dis. (in the press).

37. Doolan, D. L. et al. DNA vaccination as an approach to malaria control: current status and strategies. Curr. Top. Microbiol. Immunol. 226, 37-56 (1998).

38. Sedegah M., Hedstrom R., Hobart P. \& Hoffman S.L. Protection against malaria by immunization with plasmid DNA encoding circumsporozoite protein. Proc. Natl Acad. Sci. USA 91, 9866-9870 (1994). 
39. Doolan, D. L. et al. Circumventing genetic restriction of protection against malaria with multigene DNA immunization: $\mathrm{CD}^{+}$cell-, interferon $\gamma$-, and nitric oxide-dependent immunity. J. Exp. Med. 183, 1739-1746 (1996).

40. Schneider, J. et al. Enhanced immunogenicity for $\mathrm{CD}^{+} \mathrm{T}$ cell induction and complete protective efficacy of malaria DNA vaccination by boosting with modified vaccinia virus Ankara. Nature Med. 4, 397-402 (1998).

41. Le, T. P. et al. Safety, tolerability and humoral immune responses after intramuscular administration of a malaria DNA vaccine to healthy adult volunteers. Vaccine 18, 1893-1901 (2000).

42. Wang, R. et al. Induction of antigen-specific cytotoxic T lymphocytes in humans by a malaria DNA vaccine. Science 282, 476-480 (1998).

43. Wang, R. et al. Induction of $\mathrm{CD} 4^{+} \mathrm{T}$ cell dependent $\mathrm{CD} 8^{+}$type 1 responses in humans by a malaria DNA vaccine. Proc. Natl Acad. Sci. USA 98, 10817-10822 (2001).

44. Rogers, W. O. et al. Multi-stage multi-antigen heterologous prime boost vaccine for Plasmodium knowlesi malaria provides partial protection in rhesus macaques. Infect. Immun. 69, 5565-5572 (2001).

45. Zhong, H. et al. Induction of protective immune responses in rhesus monkey by immunization with recombinant plasmids of polyvalent epitopes of Plasmodium falciparum using cholera toxin $B$ as adjuvant. [Translation] Yi Chuan Xue Bao [Acta Genetica Sinica] 27, 966-971 (2000).

46. Ferry, G. First DNA malaria vaccine on trial in Africa. Curr. Biol. 10, R810-R811 (2000).

47. Gramzinski, R. A. et al. Malaria DNA vaccines in Aotus monkeys. Vaccine 15, 913-915 (1997).

48. Shi, Y. P. et al. Immunogenicity and in vitro protective efficacy of a recombinant multistage Plasmodium falciparum candidate vaccine. Proc. Natl Acad. Sci. USA 96, 1615-1620 (1999).

49. Aguiar, J. C. et al. Enhancement of the immune response in rabbits to a malaria DNA vaccine by immunization with a needle-free jet device. Vaccine 20, 275-280 (2001).

50. Schneider, J. et al. A prime-boost immunisation regimen using DNA followed by recombinant modified vaccinia virus Ankara induces strong cellular immune responses against the Plasmodium falciparum TRAP antigen in chimpanzees. Vaccine 19, 4595-4602 (2001)

51. Narum, D. L. et al. Codon optimization of gene fragments encoding Plasmodium falciparum merozoite proteins enhances DNA vaccine protein expression and immunogenicity in mice. Infect. Immun. 69, 7250-7253 (2001)

52. You, Z., Huang, X., Hester, J., Toh, H. C. \& Chen, S. Y. Targeting dendritic cells to enhance DNA vaccine potency. Cancer Res. 61, 3704-3711 (2001).

53. O'Hagan, D. et al. Induction of potent immune responses by cationic microparticles with adsorbed human immunodeficiency virus DNA vaccines. J. Virol. 75, 9037-9043 (2001).

54. Degano, P., Schneider, J., Hannan, C. M., Gilbert, S. C. \& Hill, A. V. Gene gun intradermal DNA immunization followed by boosting with modified vaccinia virus Ankara: enhanced $\mathrm{CD} 8^{+} \mathrm{T}$ cell immunogenicity and protective efficacy in the influenza and malaria models. Vaccine 18, 623-632 (1999).

55. Sakai, T. et al. DNA immunization with Plasmodium falciparum serine repeat antigen: regulation of humoral immune response by coinoculation of cytokine expression plasmid. Parasitol. Int. 48, 27-33 (1999)

56. Weiss, W. R. et al. A plasmid encoding murine granulocyte-macrophage colony-stimulating factor increases protection conferred by a malaria DNA vaccine. J. Immunol. 161, 2325-2332 (1998).

57. Sedegah, M. et al. Improving protective immunity induced by DNA-based immunization: priming with antigen and GM-CSF-encoding plasmid DNA and boosting with antigen-expressing recombinant poxvirus. J. Immunol. 164, 5905-5912 (2000).

58. Jones, T. R. et al. Synthetic oligodeoxynucleotides containing CpG motifs enhance immunogenicity of a peptide malaria vaccine in Aotus monkeys. Vaccine 17, 3065-3071 (1999).

59. Barouch, D. H. et al. Control of viremia and prevention of clinical AIDS in rhesus monkeys by cytokine-augmented DNA vaccination. Science 290, 486-492 (2000).

60. Schneider, J. et al. Induction of $\mathrm{CD} 8^{+} \mathrm{T}$ cells using heterologous prime-boost immunisation strategies. Immunol. Rev. 170, 29-38 (1999).

61. Jones, T. R. et al. Protection of Aotus monkeys by Plasmodium falciparum EBA-175 region II DNA prime-protein boost immunization regimen. J. Infect. Dis. 183, 303-312 (2001).

62. Dong, W. et al. Assessment of a vaccinia virus vectored multi-epitope live vaccine candidate for Plasmodium falciparum. Int. J. Parasitol. 31, 57-62 (2001).

63. Ockenhouse, C. F. et al. Phase I/IIa safety, immunogenicity, and efficacy trial of NYVAC-Pf7, a poxvectored, multiantigen, multistage vaccine candidate for Plasmodium falciparum malaria. J. Infect. Dis. 177, 1664-1673 (1998)

64. Rodrigues, E. G. et al. Interferon- $\gamma$-independent $\mathrm{CD} 8^{+} \mathrm{T}$ cell-mediated protective anti-malaria immunity elicited by recombinant adenovirus. Parasite Immunol.22, 157-160 (2000).
65. Davis, N. L. et al. Vaccination of macaques against pathogenic simian immunodeficiency virus with Venezuelan equine encephalitis virus replicon particles. J. Virol. 74, 371-378 (2000).

66. Stowers, A. W. \& Miller, L. H. Are trials in new world monkeys on the critical path for blood-stage malaria-vaccine development? Trends Parasitol. 17, 415-419 (2001).

67. Heppner, D. G. et al. New world monkey efficacy trials for malaria vaccine development: critical path or detour? Trends Parasitol. 17, 419-425 (2001).

68. Genton, B. et al. The epidemiology of malaria in the Wosera area, East Sepik Province, Papua New Guinea, in preparation for vaccine trials. II. Mortality and morbidity. Ann. Trop. Med. Parasitol. 89, 377-390 (1995).

69. Smith, T. A., Leuenberger, R. \& Lengeler, C. Child mortality and malaria transmission intensity in Africa. Trends Parasitol. 17, 145-149 (2001).

70. Church L.W. et al. Clinical manifestations of Plasmodium falciparum malaria experimentally induced by mosquito challenge. J Infect. Dis. 175, 915-920 (1997).

71. Hagan, P., Bjorvatn, B. \& Jepsen, S. European malaria vaccine initiative. Parasitol. Today 15, 47-48 (1999)

72. Nossal, G. J. The global alliance for vaccines and immunization—a millennial challenge. Nature Immunol. 1, 5-8 (2000).

73. Hoffman, S. L., Franke, E. D., Hollingdale, M. R. \& Druilhe, P. in Malaria Vaccine Development: a Multi-immune Response Approach (ed. Hoffman, S. L.) 35-75 (ASM, Washington, 1996).

74. Perlaza, B. L. et al. Immunogenicity of four Plasmodium falciparum pre-erythrocytic antigens in Aotus lemurinus monkeys. Infect. Immun. 66, 3423-3428 (1998).

75. Daubersies, P. et al. Protection against Plasmodium falciparum malaria in chimpanzees by immunization with the conserved pre-erythrocytic liver-stage antigen 3. Nature Med. 6, 1258-1263 (2000).

76. Oeuvray, C. et al. Merozoite surface protein-3: a malaria protein inducing antibodies that promote Plasmodium falciparum killing by cooperation with blood monocytes. Blood 84, 1594-1602 (1994).

77. Wang, L., Richie, T. L., Stowers, A., Nhan, D. H. \& Coppel, R. L. Naturally acquired antibody responses to Plasmodium falciparum merozoite surface protein 4 in a population living in an area of endemicity in Vietnam. Infect. Immun. 69, 4390-4397 (2001).

78. Singh, S. et al. Biochemical, biophysical and functional characterization of bacterially expressed and refolded binding domain of Plasmodium vivax Duffy binding protein. J. Biol. Chem. 276, 17111-17116 (2001).

79. Hodder, A. N., Crewther, P. E. \& Anders, R. F. Specificity of the protective antibody response to apical membrane antigen 1. Infect. Immun. 69, 3286-3294 (2001).

80. Fox, B. A., Xing, L. P., Suzue, K., Horii, T. \& Bzik, D. J. Plasmodium falciparum: an epitope within a highly conserved region of the $47-\mathrm{kDa}$ amino-terminal domain of the serine repeat antigen is a target of parasite-inhibitory antibodies. Exp. Parasitol. 85, 121-134 (1997).

81. Collins, W. E. et al. Efficacy of vaccines containing rhoptry-associated proteins RAP1 and RAP2 of Plasmodium falciparum in Saimiri boliviensis monkeys. Am. J. Trop. Med. Hyg. 62, 466-479 (2000).

82. Lyon, J. A., Thomas, A. W., Hall, T. \& Chulay, J. D. Specificities of antibodies that inhibit merozoite dispersal from malaria-infected erythrocytes. Mol. Biochem. Parasitol. 36, 77-85 (1989).

83. Saul, A. \& Miller, L. H. A Robust neutralization test for Plasmodium falciparum malaria. J. Exp. Med. 193, F51-F54 (2001).

84. O'Donnell, R. A. et al. Antibodies against MSP- $1_{19}$ are a major component of the invasion-inhibitory response in individuals immune to malaria. J. Exp. Med. 193, 1403-1412 (2001).

85. Scherf, A., Pouvelle, B., Buffet, P. A. \& Gysin, J. Molecular mechanisms of Plasmodium falciparum placental adhesion. Cell Microbiol. 3, 125-131 (2001).

86. Schofield, L. et al. Neutralizing monoclonal antibodies to glycosylphosphatidylinositol, the dominant TNF-alpha-inducing toxin of Plasmodium falciparum: prospects for the immunotherapy of severe malaria. Ann. Trop. Med. Parasitol. 87, 617-626 (1993).

87. Doolan, D. L. \& Hoffman, S. L. DNA-based vaccines against malaria: status and promise of the multistage malaria DNA vaccine operation. Int. J. Parasitol. 31, 753-762 (2001).

88. Kumar, S. et al. A multilateral effort to develop and test multi-stage, multi-gene DNA vaccines against Plasmodium falciparum malaria. Trends Parasitol. (in the press).

\section{Acknowledgements}

We thank J. Cohen, D. Doolan, J. Epstein, A. Hill, S. Jepsen, N. Tornieporth and S.

Hoffman for comments. Funding has been provided by the US Navy Bureau of Medicine, the US Army Military Infectious Disease Research Program, the Office of Naval Research, and USAID. The opinions and assertions herein are those of the authors and are not to be construed as official or as reflecting the views of the US Navy or the US Government. 